\title{
Behavioral effects of hippocampal lesions in rats with prior intraseptal injection of 6-hydroxydopamine
}

\author{
RONALD H. BAISDEN and MICHAEL L. WOODRUFF \\ Department of Anatomy, College of Medicine, East Tennessee State University \\ Johnson City, Tennessee 37601
}

\begin{abstract}
Male hooded rats were trained on a schedule of differential reinforcement of low response rates (DRL) until a stable baseline level of responding was obtained. The rats then received a bilateral intraseptal injection of an ascorbic acid solution. They were returned to the DRL schedule for 10 days, after which they received a bilateral intraseptal injection of either the ascorbic acid solution or the vehicle containing 6-hydroxydopamine (6-OHDA). After 10 additional days on the DRL schedule, a bilateral hippocampal ablation was performed on all rats. Performance on the DRL schedule was assessed, and rats that had received 6-OHDA prior to the hippocampal ablation emitted significantly fewer responses under the DRL schedule subsequent to the lesion than those that had received just the vehicle. Fluorescence microscopy reveals a substantial loss of the catecholamines in animals receiving 6-OHDA injections as compared to normals or vehicle-treated rats. The results are discussed within the context of 6-OHDA-induced denervation supersensitivity and the possible disruption of ablation-induced collateral axonal sprouting in the septum.
\end{abstract}

The results of several experiments reported during the past 20 years have supported the hypothesis that, following trauma, the central nervous system (CNS) is capable of morphological reconstruction. Collateral sprouting is one such reconstructive change and is said to have occurred when intact axons which terminate in close proximity to an area of lesion-induced denervation appear to form new synaptic contacts with the denervated synaptic sites. Collateral axonal sprouting has been demonstrated in several regions of the CNS, including the dorsal spinal cord (Liu \& Chambers, 1958), the ventral lateral geniculate nucleus (Goodman \& Horel, 1966), the hippocampal formation (Lynch, Matthews, Mosko, Parks, \& Cotman, 1973), and the septal area (Raisman, 1969).

Although there is some evidence that the synapses formed by collateral sprouting are physiologically functional (Lynch, Deadwyler, \& Cotman, 1973), there is debate concerning the possible effect of these new connections on behavior. Raisman (1969) argued that if lesion-induced collateral axonal sprouting produced any effect on behavior, the effect would be disruptive because abnormal synaptic connections would be established which would lead to "functional confusion." Indeed, McCouch, Austin, Liu, and Liu (1958) had presented evidence which suggested that

This research was supported in part by Biomedical Research Development Grant 1-508-RR09171-01. Please address reprint requests to Ronald H. Baisden, East Tennessee State University, College of Medicine, Department of Anatomy, Box 19960A, Johnson City, Tennessee 37601. spasticity following spinal cord hemisection in the cat was due to collateral axonal sprouting, and this experiment supports Raisman's contention.

More recently, however, Steward and his co-workers presented evidence that collateral sprouting may provide the basis for recovery of behavioral function (Loesche \& Steward, 1977; Steward, Loesche, \& Horton, 1977). These researchers observed that the ability of rats to perform a reinforced alternation was impaired following a unilateral entorhinal cortex lesion, but that this impairment disappeared over time. The recovery of behavioral function was apparently due to the formation of new synaptic contacts between neurons of the dentate gyrus ipsilateral to the lesion and afferents from the contralateral entorhinal cortex. Recovery of alternation was prevented if the contralateral entorhinal cortex was ablated, or if the dorsal psalterium was transected.

In the experiments of Steward and his co-workers, the observed sprouting was of axons from the contralateral homologue of the lesioned structure. As these authors point out, the homotypic nature of the sprouting axons could have contributed to the observed recovery of behavioral function. Raisman (1969) found that destruction of the hippocampal afferents to the septal area induced sprouting of afferents reaching the septal area through the medial forebrain bundle (MFB). When compared to the hippocampal afferents, the MFB septal input is heterotypic in terms of synaptic morphology, locus of termination on septal neurons, and, presumably, of neurotransmitter content (Raisman, 1969). Because of the heterotypic 
nature of the sprouting MFB axons, it may be that, rather than aiding in recovery of function, the new synapses formed by these axonal sprouts contribute to the behavioral deficits seen after hippocampal ablation. This possibility was examined in the present experiment.

At least some of the sprouting MFB septal afferents are derived from the adrenergic component of the MFB (Moore, Bjorklund, \& Stenevi, 1971). The neurotoxin 6-hydroxydopamine (6-OHDA) can destroy selectively the catecholamine terminals within the septum (Hökfelt \& Ungerstedt, 1973). Therefore, if 6-OHDA is injected into the septum before a hippocampal lesion, much of the septal MFB projection should be destroyed and the sprouting response that is usually induced by the hippocampal lesion should be disrupted. If, then, MFB sprouting in the septal region is responsible for some of the deficits seen after hippocampal destruction, treatment of the septal area with 6-OHDA should attenuate the lesion-induced deficit. Hippocampal lesions are known to produce deficits in the performance of operant tasks which require the animal to withhold responses for some specified period of time in order to achieve a maximum density of reinforcement (differential reinforcement of low response rates, DRL; Clark \& Isaacson, 1965). This task was chosen as the behavioral paradigm in this experiment.

\section{METHOD}

\section{Subjects and Preoperative Training}

Fourteen male Long-Evans hooded rats $(300-350 \mathrm{~g})$ were used. They were housed individually under a 12-h-on/12-h-off lightdark cycle. Water was available in the home cage throughout the experiment.

Prior to testing, the subjects were deprived of food and maintained on a regimen that kept them at $85 \%$ of their ad-lib weights. All behavioral testing was done in a Lehigh Valley operant chamber (Model 1417). Electromechanical relays, counters, and timers controlled the recording of responses and presentation of reinforcement. Each rat was run for 30 min 5 days a week. The rats were shaped to press the bar and were then placed on a continuous reinforcement schedule for from 8 to 14 days, at which time a DRL 20-sec schedule was put into effect. The rats remained on the DRL schedule until they were responding at a steady rate and had achieved a consistent level of reinforced responses $( \pm 10 \%)$ over 10 days. Total presses and number of reinforcements earned in the 30-min session were tabulated for each day.

\section{Surgical Procedures}

Subsequent to development of a stable pattern of performance, the rats were divided into two groups of seven on the basis of their preoperative DRL performance such that the groups did not differ in mean response rate and reinforcement frequency.

The following operations were performed. Each rat was anesthetized with sodium pentobarbital (Nembutal, $50 \mathrm{mg} / \mathrm{kg}$, i.p.). Its scalp was shaved and it was placed in a Kopf stereotaxic instrument. The dorsum of the skull was exposed by a midline incision and removal of the scalp muscles and fascia. Bilateral burr holes were produced $2.0 \mathrm{~mm}$ anterior to bregma, and rongeurs were used to connect these holes across the midline, thereby exposing the dura and superior midsagittal sinus. A cannula constructed of 22-ga hypodermic tubing was then lowered into the septal area on one side. After the injection procedure described below was completed, this cannula was raised and a second cannula was lowered into the contralateral septum. The anterior and ventral coordinates were +2.0 and $-5.0 \mathrm{~mm}$, respectively (Pellegrino \& Cushman, 1967). Lateral placements varied slightly with the course of the sinus, but were approximately $\pm .7 \mathrm{~mm}$ from the midline of the brain.

After the 22-ga cannula was in place, a 27-ga hypodermic tube was inserted into it. After insertion, this inner cannula extended approximately $1.0 \mathrm{~mm}$ beyond the tip of the outer cannula. It was connected to a Hamilton microliter syringe by P.E. 50 intramedic tubing. This entire system had been filled with a solution composed of $1.0 \mathrm{mg} / \mathrm{ml}$ ascorbic acid in $.85 \%$ aqueous solution. This solution served as the vehicle for the 6-OHDA (Hedreen \& Chalmers, 1972), and its effects on DRL performance were determined for all rats before any subjects received the 6-OHDA solution.

Four microliters of the ascorbic acid vehicle were injected into each half of the septum at a rate of $.3 \mu \mathrm{l} / \mathrm{min}$. The rate and volume of injection were controlled by a Sage Instruments syringe pump (Model 341). Hemostasis was obtained upon completion of the injection procedure, and the scalp was closed. Bicillin $(25,000$ units i.m.) was given to combat possible postoperative infection. This amount of Bicillin was also given after each of the operations described below.

After a 2-day recovery period, the animals were returned to the DRL schedule and tested for 10 days. Thirteen days after the first vehicle control injection had been made, seven rats received another vehicle injection. These rats formed the vehicle control group. On the same day, the other seven rats received the vehicle containing $2.0 \mu \mathrm{g} / \mu \mathrm{l}$ 6-OHDA HBr (Sigma Chemical). These animals comprised the experimental group. All injection solutions were prepared just prior to use and stored under refrigeration until used. If, during use, the solution became discolored, it was discarded and a fresh sample prepared. The anesthetization and operative procedures were similar to those described above.

Two days following the second operation, the subjects were placed back on the DRL schedule and tested for 10 days. Bilateral hippocampal aspirations were performed on all subjects 13 days after the second intraseptal injection. The procedure for producing these lesions has been described in detail elsewhere (Isaacson \& Woodruff, 1976). A 5-day recovery period was allowed, after which the rats were returned to the DRL regimen for an additional 10 days. One rat from the vehicle-injected control group died following the hippocampal lesion.

\section{Histology}

At the conclusion of behavioral testing, all operated rats were killed with an overdose of Nembutal and intracardially perfused with $.9 \%$ saline followed by $10 \%$ Formalin. The brains were removed, embedded in celloidin, and sectioned at $30 \mu \mathrm{m}$. Every section through the septum and every fifth section through the hippocampus was retained, slide mounted, and stained with thionin.

Because the requisite equipment was not available at the time that the behavioral assessment had to be terminated, the brains of the rats tested on the DRL schedule could not be processed such that any estimation of the effectiveness of 6-OHDA in depleting catecholamines in the septal area could be made. Subsequently, however, a fluorescent microscope was obtained and the SPG histofluorescent procedure of de la Torre and Surgeon (1976), as modified by de la Torre (1979), was introduced into our laboratory. This procedure was used to evaluate the pattern of catecholaminergic innervation of the septal area in six rats. Two of these rats were unoperated. Two rats were given intraseptal 6-OHDA injections, and two rats were given intraseptal vehicle injections. The rats receiving operations were allowed 6 weeks for postoperative recovery. All six rats were killed by decapitation with a 
guillotine, and the unfixed brains were immediately removed and frozen in a cryostat. Thirty-micron sections were taken through the septal area and hippocampus. These sections were processed by the SPG procedure for histofluorescence.

\section{RESULTS}

\section{Histology}

Examination of the histology confirmed that bilateral injections had been made into the septal area in all but one rat. This rat had received 6-OHDA, and on one side the cannula had penetrated into the lateral ventricle. Data from this rat were not included in subsequent analyses. In the other rats, the cannula had penetrated the lateral septal nucleus and the cannula tracks ended either in this nucleus or in the lateral aspect of the medial septal nucleus.

Photomicrographs of representative thionin-stained sections of the septal area from rats in the vehicle control and 6-OHDA-injected groups are presented in Figure 1. As can be seen in this figure, there was no sign in the thionin-stained sections that local in-

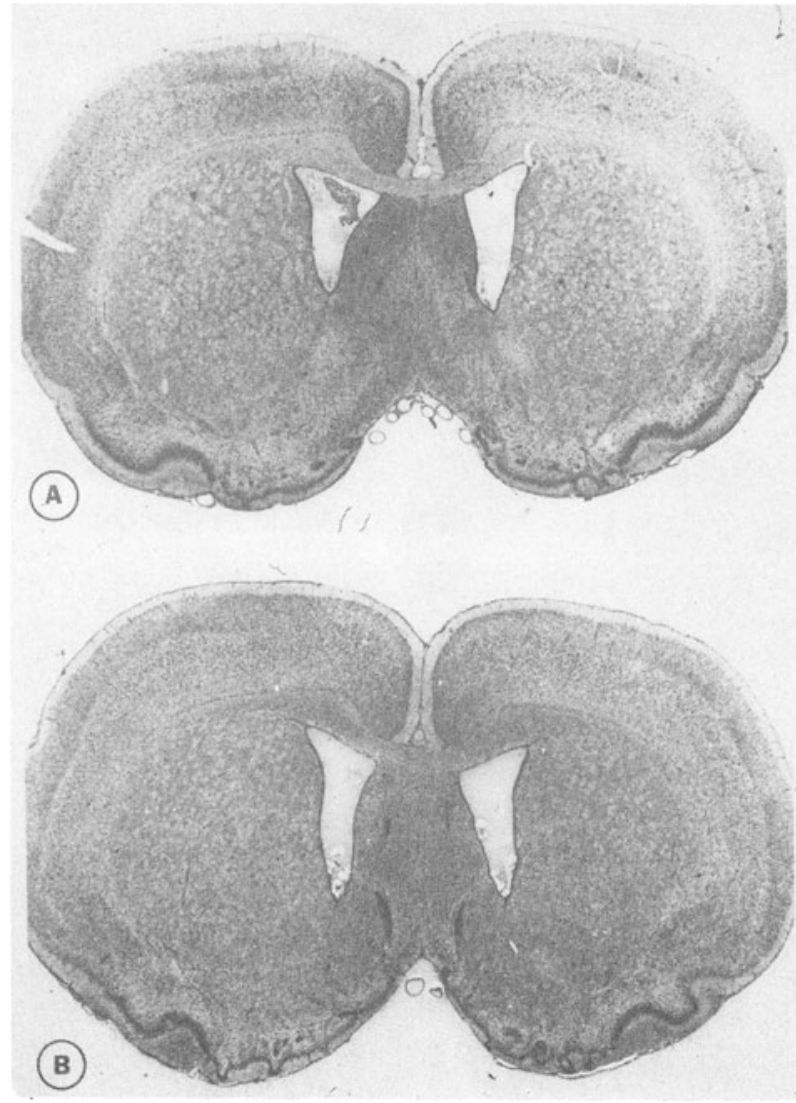

Figure 1. Representative thionin-stained sections from the septal area of subjects in the 6-OHDA-injected group (A) and the vehicle control group (B).
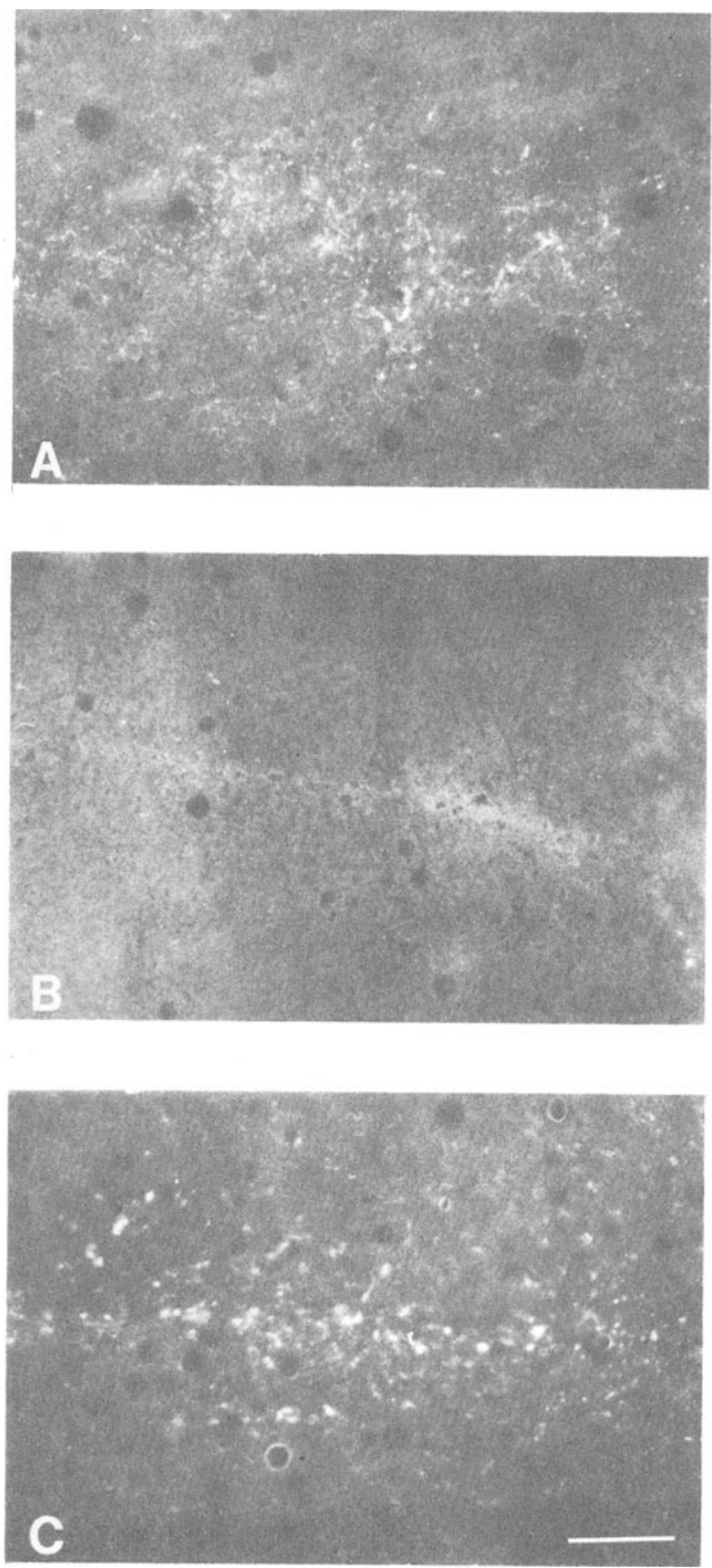

Figure 2. Glyoxylic acid-induced histofluorescence in the septal region of an unoperated rat, a rat given bilateral intraseptal injections of 6-OHDA, and a rat given bilateral intraseptal injections of the vehicle solution. (A) Terminal patch in the lateral septal nucleus of an untreated rat; (B) cannula track through the septum of an animal with local injection of 6-OHDA; and (C) cannula track after vehicle injection into septal region. The increased size of the granules observed in $C$, as compared to $A$, appears to be due to swelling produced by nonspecific damage from the injection. Bar $=100 \mu \mathrm{m}$. 
jections of 6-OHDA produced tissue damage in excess of that produced by the vehicle control injections. This observation is in disagreement with some earlier reports (Poirer, Langelier, Robeege, Boucher, \& Kitsikis, 1972). In the present experiment, neuron loss and gliosis in the septum were observed only around the cannulae tracks in both vehicle- and 6-OHDA-injected rats.

Fluorescence microscopy, however, revealed substantial loss of monoamine innervation throughout the septal region in 6-OHDA treated rats. Monoaminecontaining terminals were clearly present in the vehicletreated animals (Figure 2). After drug injections, the loss appeared to be limited to the region of the septum, and included the normally dense catecholamine terminals in the nucleus accumbens. In these animals, a normal-appearing catecholaminergic innervation was observed in the striatum, cerebral neocortex, and hippocampus (Figure 3).

Reconstructions and photomicrographs of the
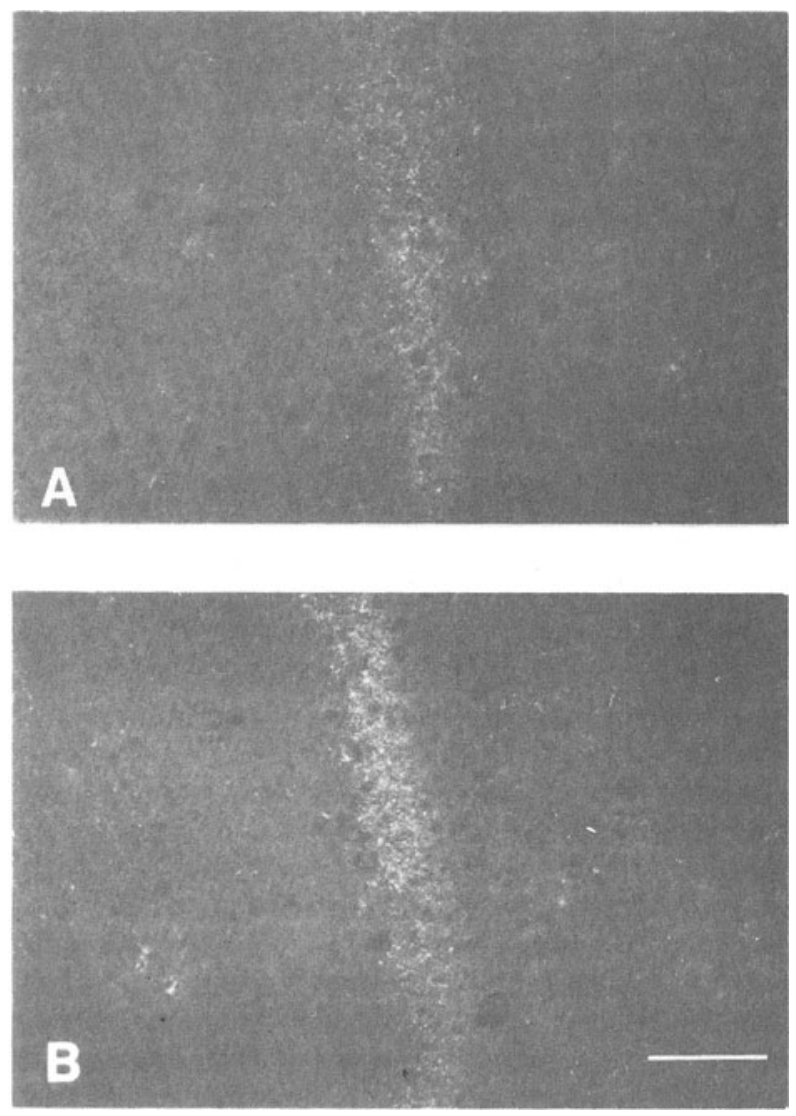

Figure 3. Fluorescing terminals in the pyramidal layer of the hippocampal subfield $\mathrm{CA}_{1}$ at the septal end of the dorsal hippocampus. No apparent differences exist between the normal rat (A) and the rat that received an intraseptal 6-OHDA injection (B). This indicates that the effect of the neurotoxin was relatively restricted to the area of injection. Bar $=100 \mu \mathrm{m}$.

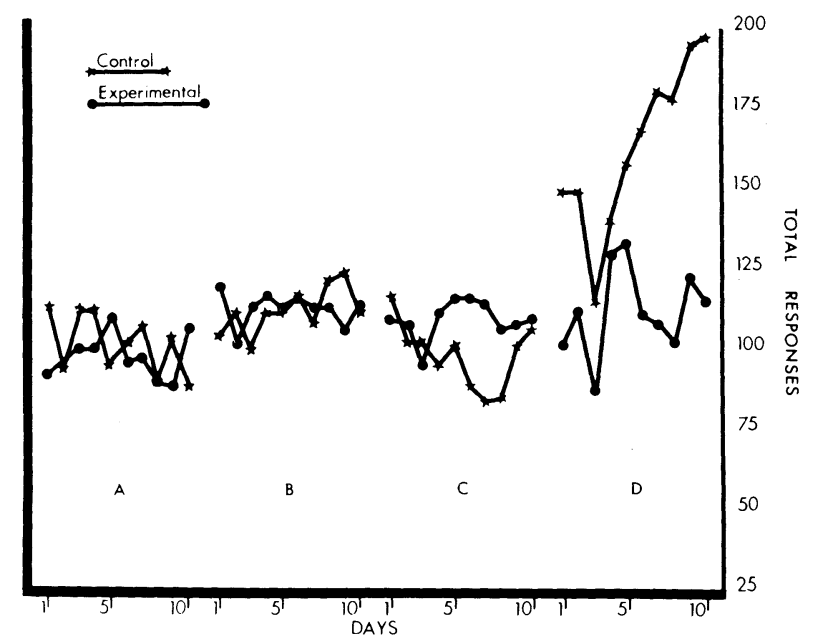

Figure 4. Mean number of responses for each group for the preoperation period $(A)$; the period after the intraseptal vehicle injection (B); the period following the second intraseptal injection (C); and the period following the hippocampal ablation (D).

aspiration-produced lesions of the hippocampus revealed damage similar to that observed in previous studies done in this laboratory (Woodruff \& Isaacson, 1972). There was virtually complete removal of the dorsal and middle portions of the hippocampus. A varying amount of ventral hippocampus was spared. Extrahippocampal damage included the overlying neocortex and corpus callosum.

\section{Behavioral Sequelae}

The mean number of responses for each group under the DRL schedule for the final 10 days before any operations were performed, for the 10 days of testing subsequent to each intraseptal injection, and for the 10 days of testing following hippocampal lesion are presented in Figure 4. A repeated measures analysis of variance (Winer, 1971) was performed for each of these blocks of days. There were no statistically significant differences between groups in the preoperative condition or after either intraseptal injection. A significant group effect was found for the 10 test days subsequent to the hippocampal lesion $(\mathrm{F}=5.03, \mathrm{df}=1,10, \mathrm{p}<.05)$ and a significant days effect also emerged $(F=2.93, \mathrm{df}=9,90, \mathrm{p}<.01)$. The Group by Days interaction did not achieve statistical significance $(\mathrm{F}=1.74, \mathrm{df}=9,90, \mathrm{p}<.10)$.

The mean number of reinforcements earned during the final 10 preoperative days, the 10 days of testing subsequent to each intraseptal injection, and for the 10 days of testing following hippocampal lesion, are presented for each group in Figure 5. A repeated measures analysis of variance was performed for each of these blocks of days. There were no statistically significant differences between groups in the preoperative condition or after either intraseptal in- 
jection. No significant group effect was found for the test days following hippocampectomy $(F=2.00$, $\mathrm{df}=1,10, \mathrm{p}<.10$ ), nor was the days effect significant $(\mathrm{F}=0.44, \mathrm{df}=9,90, \mathrm{p}<.10)$, but a significant Group by Days interaction was found for this test condition $(F=2.44, \mathrm{df}=9,90, \mathrm{p}<.02)$.

The mean percent of correct responses made by each group during each of the test periods is presented in Figure 6. There were no statistically significant differences between groups in the preoperative condition or after either intraseptal injection. A significant group effect was found after the hippocampal lesion $(\mathrm{F}=6.66, \mathrm{df}=1,10, \mathrm{p}<.05)$, but neither the days effect nor the Groups by Days interaction was significant $(F=1.11, d f=9,90, p>.10$; $F=1.58, \mathrm{df}=9,90, \mathrm{p}>.10)$.

\section{DISCUSSION}

The principal result of this experiment is that rats given a bilateral intraseptal injection of 6-OHDA 13 days before receiving a bilateral hippocampal ablation responded at significantly lower rates on a DRL schedule after the ablation than rats that received only a preablation vehicle injection. There were no statistically significant differences between the groups in the number of reinforcements earned after the ablation, although increased response rate by the control group after ablation caused the performance of this group to be less efficient than that of the experimental group as reflected in a significantly lower percentage of reinforcement.

Although neither a significant group effect nor a significant days effect emerged for number of rein-

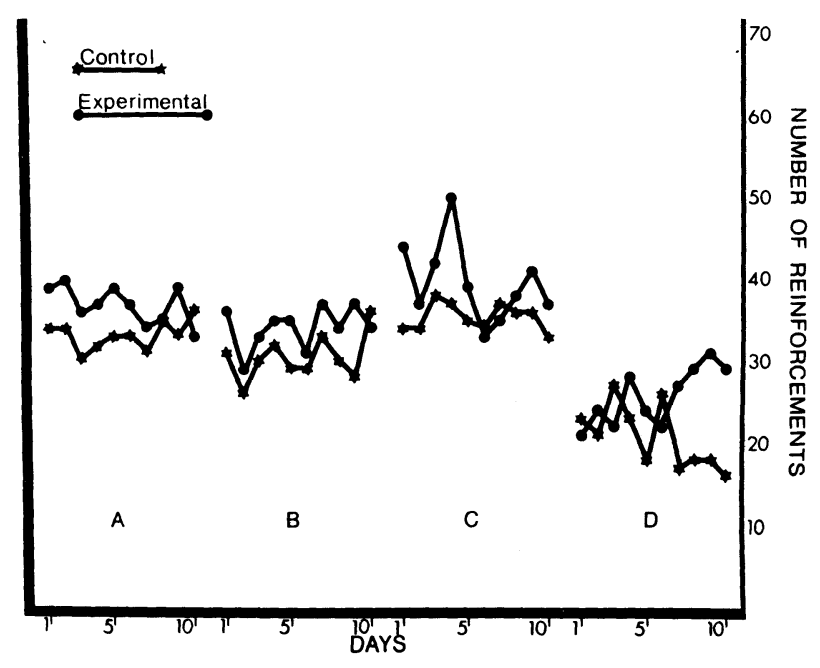

Figure 5. Mean number of reinforcements for each group for the preoperation period (A); the period after the intraseptal vehicle injection (B); the period following the second intraseptal injection(C); and the period following the hippocampal ablation (D).

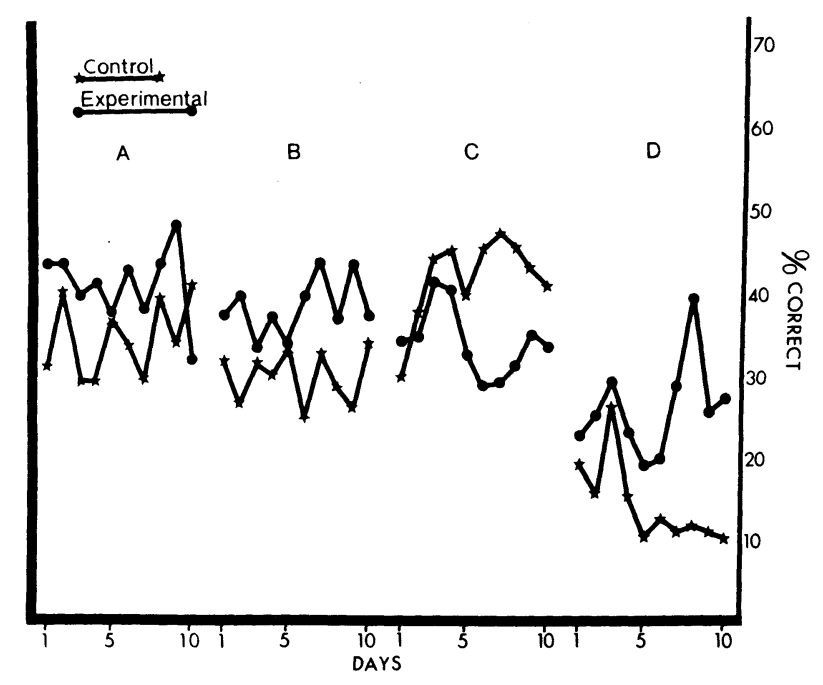

Figure 6. Mean percent correct for each group for the preoperation period $(A)$; the period after the intraseptal vehicle injection (B); the period following the second intraseptal injection (C); and the period following the hippocampal ablation (D).

forcements earned following the hippocampal ablation, a significant Group by Days interaction was found. From inspection of Figure 5 it appears that the rats in the experimental group increased the number of reinforcements that they earned over test sessions, whereas the number of reinforcements earned by the rats in the control group decreased. This would account for the significant interaction effect.

It should also be noted that a significant days effect was found for the total number of responses emitted after the lesion. While the Days by Groups interaction for total responses did not achieve putative levels of statistical significance, Figure 4 suggests that the number of responses was increasing more rapidly for the control group than for the experimental group during the final 5 days of testing. Taken together, the data concerning number of reinforcements earned and total responses emitted following the hippocampal ablation indicate that the 6-OHDA had more of an effect upon response rate than upon the ability of the rats to respond to the contingency of the schedule after receiving a hippocampal lesion. Although the rats in the control group emitted significantly more responses than the experimental animals over the 10 days of postablation testing, they apparently were able to time these responses such as to obtain about the same number of reinforcements as the experimental group. These data suggest that more than one neural mechanism is involved in the regulation of DRL performance in the rat and that damage to the hippocampo-septal circuit may lead to overresponding without simultaneous loss of the ability to respond to the contingencies of 
the schedule. Ellen, Makohon, and Richardson (1978) have argued for this position and have presented data which support it.

Subsequent to 6-OHDA injection, a loss of monoamine fluorescence was observed throughout the septum. The monoaminergic innervation of the hippocampus and cerebral neocortex appeared to be similar to that found in normal rats (Figure 3). This suggests that the intraseptal injections of 6-OHDA did produce selective destruction of catecholaminergic septal afferents. Moreover, it would appear that this destruction attenuated the deficit in DRL performance observed in the vehicle-injected control rats following hippocampal ablation.

At least two explanations are possible for these results. The first of these is that the 6-OHDA-induced loss of septal afferents resulted in development of denervation supersensitivity. Partial denervation often results in increases in both the excitability and sensitivity of neurons to remaining afferent input (Sharpless, 1969). It has been proposed that denervation-induced changes in the response characteristics of neurons (e.g., supersensitivity) may account for the reduction over time of some lesion effects. For example, Berger, Wise, and Stein (1971) suggested that recovery from lateral hypothalamic damage might be attributable to denervation supersensitivity. Experiments by Balagura, Harrell, and Ralph (1973) and Glick, Greenstein, and Zimmerberg (1972) demonstrated that pretreatment with drugs that have effects similar to 6-OHDA reduced the duration of the recovery time following lateral hypothalamic lesions.

DRL performance in the absence of an external cue is impaired following a septal lesion (Ellen, Wilson, \& Powell, 1964). Integrity of this structure is, therefore, at least as important for performance of the DRL task as integrity of the hippocampus. If septal neurons develop denervation supersensitivity after 6-OHDA treatment, then it is possible that this supersensitivity compensates for reduction in hippocampal influence after the lesion. This is especially likely if, as was the case in the present experiment, less than total removal of the hippocampus had occurred. The hippocampal efferents remaining to the septum could have exerted sufficient influence on supersensitive septal neurons to compensate for a reduction in the number of septal inputs. Since the septal neurons of the vehicle-injected controls were not denervated, they presumably were not supersensitive to hippocampal input and, therefore, could not compensate for reduction in hippocampal afferents after the ablation.

A second explanation for the results obtained in this experiment is presented in the hypothesis offered in the introduction. By destroying many of the septal MFB afferents, the 6-OHDA treatment would presumably reduce the number of MFB axons which would develop collateral sprouts in response to the hippocampal ablation. Therefore, the "functional confusion" which Raisman (1969) proposed would occur as a result of such axonal sprouting would be eliminated or reduced. Implicit in this hypothesis is the idea that the deficit observed in DRL performance following hippocampal ablation (Clark \& Isaacson, 1965) is not due directly to removal of hippocampal influence, but, rather, is due to collateral sprouting of MFB afferents in the septal area.

If this hypothesis is valid, then a parallel temporal relationship should exist between the hippocampal lesion-induced increase in response rate on the DRL schedule and catecholaminergic sprouting in the septal area. Moore et al. (1971, p. 21) have reported that only "a suggestive increase in catecholaminergic terminals" is observed in the septal area 8 days after anterior hippocampal destruction and that the septal area does not demonstrate a substantial increase above normal levels in catecholaminergic histofluorescence until 15 days after the ablation. In the present study, the lesion-induced increase in response rate on the DRL schedule appears to precede the time required for obvious axonal sprouting in the septal area in that rats given hippocampal lesions without prior 6-OHDA treatment overresponded 6 days after the ablation. However, closer examination of the data obtained in this experiment suggests that the time course of septal sprouting might indeed be related to the higher than normal response rate produced by rats with hippocampal destruction performing under the DRL contingency. The number of responses for the group of rats with hippocampal ablation and no intraseptal 6-OHDA demonstrated a statistically significant increase from Postablation Day 6 through the final day of testing, which was Postablation Day 15 (Figure 4). This period corresponds to the posthippocampal ablation time span when Moore et al. (1971) found that the amount of septal catecholaminergic histofluorescence increased to a level which noticeably exceeded that found in the unablated condition. The data obtained from the behavioral measurements made in the present experiment do not, therefore, entirely fail to indicate a correspondence between the time course for the development of overresponding on the DRL schedule by rats with hippocampal lesions and hippocampal lesioninduced increases in catecholaminergic innervation of the septal area. The increase in response rate observed before Postablation Day 9 may be due either to catecholaminergic sprouting which is not detectable using histofluorescent techniques or to some such process as denervation supersensitivity. Lesioninduced supersensitivity may occur, at least in the partially denervated hippocampus, as early as 2 days after the lesion (Bird \& Aghajanian, 1975).

If hippocampal lesion-induced axonal sprouting in 
the septal area is responsible, at least in part, for postlesion response rate increases, this suggests that collateral sprouting in a heterotypic system may lead to behavioral dysfunction. Previous work by Loesche and Steward (1977) and Steward et al. (1977) indicates that lesion-induced collateral sprouting in a homotypic system may lead to recovery of behavioral function. Taken together, these observations suggest that whether or not collateral axonal sprouting leads to behavioral recovery may depend upon whether sprouting axons are derived from homotypic or heterotypic sources.

Either 6-OHDA-induced supersensitivity or prevention of collateral sprouting in the septal area may be responsible for the results obtained in this experiment. Some combination of these effects of the intraseptal 6-OHDA injection also may have led to the observed reduction in DRL impairment following the hippocampal ablation. Definitive evidence in support of one of these alternatives, to the exclusion of the others, is not presently available. However, as pointed out by Schoenfeld and Hamilton (1977), secondary effects of brain lesions, such as collateral sprouting and supersensitivity, may be the changes which are important in producing behavioral changes subsequent to brain lesions. The data from this experiment support the contention that changes (functional and/or morphological) in a structure that is not damaged directly by a lesion produce behavioral effects.

\section{REFERENCES}

Balagura, S. L., Harrell, L., \& Ralph, T. Glucodynamic hormones modify the recovery period after lateral hypothalamic damage. Science, 1973, 182, 59-60.

Berger, B. D., Wise, C. D., \& Stein, L. Norepinephrine: Reversal of anorexia in rats with lateral hypothalamic damage. Science, 1971, 172, 281-284.

Bird, S. J., \& Aghajanian, G. K. Denervation super-sensitivity in the cholinergic septo-hippocampal pathway: A microiontophoretic study. Brain Research, 1975, 100, 355-370.

Clark, C. V. H., \& IsaAcson, R. L. Effect of bilateral hippocampal ablation on DRL performance. Journal of Comparative and Physiological Psychology, 1965, 59, 137-140.

de la Torre, J. C., \& Surgeon, J. W. A methodological approach to rapid and sensitive monoamine histofluorescence using a modified glyoxylic acid technique: The SPG method. Histochemistry, 1976, 49, 81-93.

DE LA TORRE, J.C. Standardization of the SPG histofluorescence method for monoamine neurotransmitters. Society for Neurosciences Abstracts, 1979, 5, 333.

Ellen, P., Makohon, L., \& Richardson, W. K. Response suppression on DRL by rats with septal damage. Journal of Comparative and Physiological Psychology, 1978, 92, 511-521.

Ellen, P., Wilson, A. S., \& Powell, E. W. Septal inhibition and timing behavior. Experimental Neurology, 1964, 10, 120-132.
Glick, S. D., Greenstein, S., \& Zimmerberg, B. Facilitation of recovery by alpha-methyl-para-tyrosine after lateral hypothalamic damage. Science, 1972, 177, 534-535.

Goodman, D. C., \& Horel, J. A. Sprouting of optic tract projections in the brain stem of the rat. Journal of Comparative Neurology, 1966, 127, 71-88.

Hedreen, J. G., \& Chalmers, I. P. Neuronal degeneration in rat brain by 6 -hydroxydopamine; a histological and biochemical study. Brain Research, 1972, 47, 1-36.

HökFELT, T., \& UNGERSTEDT, U. Specificity of 6-hydroxydopamine induced degeneration of central monoamine neurons: An electron and fluorescence microscopic study with special reference to intracerebral injection on the nigrostriatal dopamine system. Brain Research, 1973, 60, 269-297.

IsaACSon, R. L., \& WoodrufF, M. L. Spontaneous alternation and passive avoidance behavior in rats after hippocampal lesions. In B. Hart (Ed.), Experimental psychobiology: A laboratory manual. San Francisco: Freeman, 1976.

Liv, C. N., \& Ch AmBers, W. W. Intraspinal sprouting of dorsal root axons. Archives of Neurology and Psychiatry, 1958, 79, 46-61.

Loesche, J., \& Steward, O. Behavioral correlates of denervation and reinnervation of the hippocampal formation of the rat: Recovery of alternation performance following unilateral entorhinal cortex lesions. Brain Research Bulletin, 1977, 2, 31-39.

Lynch, G., Deadwyler, S., \& Cotman, C. Postlesion axonal growth produces permanent functional connections. Science, 1973, 180, 1364-1366.

Lynch, G., Matthews, D. A., Mosko, S., Parks, T., \& Cotman, C. C. Induced ACHE-rich layer in rat dentate gyrus following entorhinal lesions. Brain Research, 1973, 42, 311-318.

McCouch, G. P., Austin, G. M., Liv, C. N., \& Liv, C. Y. Sprouting as a cause of spasticity. Journal of Neurophysiology, 1958, 21, 205-216.

Moore, R. Y., Buorklund, A., \& Stenevi, U. Plastic changes in the adrenergic innervation of the rat septal area in response to denervation. Brain Research, 1971, 33, 13-35.

Pellegrino, L. J., \& Cushman, A. J. A stereotaxic atlas of the rat brain. New York: Appleton-Century-Crofts, 1967.

Poirer, L. S., Langelier, P., Robeege, A., Boucher, R., \& KITSIKIS, A. Nonspecific histopathological changes induced by intracerebral injection of 6-hyroxydopamine (6-OHDA). Journal of Neurological Sciences, 1972, 16, 401-416.

RAISMAN, G. Neuronal plasticity in the septal nuclei of the adult rat. Brain Research, 1969, 14, 25-48.

Schoenfeld, T. A., \& Hamilton, L. W. Secondary changes following lesions: A new paradigm for lesion experimentation. Physiology \& Behavior, 1977, 18, 951-967.

Sharpless, S. K. Isolated and deafferented neurons: Disuse supersensitivity. In H. H. Jasper, A. A. Ward, \& A. Pope (Eds.), Basic mechanisms of the epilepsies. Boston: Little, Brown, 1969.

Steward, O., Loesche, J., \& Horton, W. C. Behavioral correlates of denervation and reinnervation of the hippocampal formation of the rat: Open field activity and cue utilization following bilateral entorhinal cortex lesions. Brain Research Bulletin, 1977, 2, 41-48.

WINER, B. J. Statistical principles in experimental design. New York: McGraw-Hill, 1971.

WoodrufF, M. L., \& IsaAcson, R. L. Discrimination learning in animals with lesions of hippocampus. Behavioral Biology, 1972, 7, 489-501.

(Received for publication August 6, 1979; revision accepted January 9,1980 .) 